\title{
Nonoscillatory and Oscillatory Criteria for First Order Nonlinear Neutral Impulsive Differential Equations
}

\author{
I. O. Isaac \\ Department of Mathematics/Statistics and Comp. Science, University of Calabar \\ P.M.B. 1115, Calabar, Cross River State, Nigeria \\ E-mail: idonggrace@yahoo.com \\ Z. Lipcsey \\ Department of Mathsematics/Statistics and Comp. Science, University of Calabar \\ P.M.B. 1115, Calabar, Cross River State, Nigeria \\ E-mail: zlipcsey@yahoo.com \\ U. J. Ibok \\ Department of Pure and Applied Chemistry, University of Calabar \\ P.M.B. 1115, Calabar, Cross River State, Nigeria \\ E-mail: ibokuj@yahoo.com
}

Received: January 11, 2011 Accepted: January 26, 2011 doi:10.5539/jmr.v3n2p52

\begin{abstract}
A survey of recent studies in neutral impulsive differential equations reveals that most of such works revolve around the quest for oscillatory conditions for linear impulsive differential equations. The development of oscillatory and nonoscillatory criteria for nonlinear impulsive differential equations has so far attracted very little attention. In this paper, we obtain sufficient conditions for the existence of oscillatory and nonoscillatory solutions for nonlinear first order neutral impulsive differential equations with constant delays.
\end{abstract}

Keywords: Nonlinear neutral impulsive differential equations, Nonoscillatory and Oscillatory conditions

\section{Introduction}

Research about oscillations for linear neutral impulsive differential equations with or without delays has enjoyed unprecedented patronage in recent times (Isaac and Lipcsey, 2009b, c; Isaac and Lipcsey, 2010a, b; Graef et al, 2002; Graef et al, 2004; El-Morshedy and Gopalsamy, 2000; Luo et al, 2000; Giang and Gyori, 1993). Unfortunately, there appear to be limited investigations about oscillations for nonlinear neutral impulsive differential equations which underline the foundation of modern applications. Even the limited studies are mainly concerned with linearization techniques (Isaac and Lipcsey, 2009a; Berezansky and Braverman, 1996). Worse still, the concept of nonoscillations for nonlinear neutral impulsive equations presently suffers almost complete neglect. In this study, we make a deliberate attempt to clear these obstacles and extend the concepts beyond the existing boundaries.

We begin with the discussion on the existence of nonoscillatory solutions for first order nonlinear neutral impulsive differential equations

$$
\left\{\begin{array}{l}
{\left[y(t)-\sum_{i=1}^{m} p_{i}(t) y\left(t-\tau_{i}\right)\right]^{\prime}+f\left(t, y\left(t-\sigma_{1}\right), \cdots, y\left(t-\sigma_{n}\right)\right)=0, \quad t \neq t_{k}} \\
\Delta\left[y\left(t_{k}\right)-\sum_{i=1}^{m} p_{i}\left(t_{k}\right) y\left(t_{k}-\tau_{i}\right)\right]+g\left(t_{k}, y\left(t-\sigma_{1}\right), \cdots, y\left(t_{k}-\sigma_{n}\right)\right)=0, \quad \forall t=t_{k}
\end{array}\right.
$$

for $t \geq t_{0}>0$ and $k: t_{k} \geq t_{0}>0$ identifying some essential sufficient criteria. Next, we study the oscillations of the nonlinear neutral impulsive equation

$$
\begin{cases}\left.\left[y(t)+P(t) y\left(t-\tau_{i}\right)\right]^{\prime}+\left.q(t)\left[\prod_{\ell=1}^{m_{j}} \mid y(t)-\sigma_{\ell}\right)\right|^{\alpha_{\ell}}\right] \operatorname{sign}(y(t))=0 & t \neq t_{k} \\ \left.\Delta\left[y\left(t_{k}\right)+P\left(t_{k}\right) y\left(t_{k}-\tau_{i}\right)\right]+\left.q_{k}\left[\prod_{\ell=1}^{m_{j}} \mid y\left(t_{k}\right)-\sigma_{\ell}\right)\right|^{\alpha_{\ell}}\right] \operatorname{sign}\left(y\left(t_{k}\right)\right)=0, & \forall t=t_{k}\end{cases}
$$

obtaining some new conditions for all solutions of Equation (1.2) to be oscillatory. 
Our conditions are "sharp" in the following sense. If Equations (1.1) and (1.2) are linear with constant coefficients, the conditions become both necessary and sufficient. In what follows, we recall some of the basic notions and definitions that will be of importance as we advance through the article.

The solution $y(t)$ for $t \in\left[t_{0}, T\right)$ of a given impulsive differential equation or its first derivative $y \prime(t)$ is a piece-wise continuous function with points of discontinuity $t_{k} \in\left[t_{0}, T\right), t_{k} \neq t, 0 \leq k<\infty$. Consequently, in order to simplify the statements of our assertions later, we introduce the set of functions $P C$ and $P C^{r}$ which are defined as follows:

Let $r \in N, D:=[T, \infty) \subset R$ and let the set $S:=\left\{t_{k}\right\}_{k=N}$ be fixed. Except stated otherwise, we will assume that the elements of $S$ are moments of impulse effect and satisfy the property:

C1.1 $0<t_{1}<t_{2}<\cdots$ and $\lim _{k \rightarrow+\infty} t_{k}=+\infty$.

We denote by $P C(D, R)$ the set of all functions $\varphi: D \rightarrow R$, which are continuous for all $t \in D, t \notin S$. They are continuous from the left and have discontinuity of the first kind at the points for which $t \in S$, while by $P C^{r}(D, R)$, we denote the set of functions $\varphi: D \rightarrow R$ having derivative $\frac{d^{j} \varphi}{d t^{j}} \in P C(D, R), 0 \leq j \leq r$ (Bainov and Simeonov, 1998).

To specify the points of discontinuity of functions belonging to $P C$ or $P C^{r}$, we shall sometimes use the symbols $P C(D, R ; S)$ and $P C^{r}(D, R ; S), r \in N$.

\section{Definition 1.1}

A solution $y(t)$ of Equation (1.1) or (1.2) is said to be

(i) Finally positive (finally negative) if there exists $T \geq 0$ such that $y(t)$ is defined and is strictly positive (strictly negative) for $t \geq T$;

(ii) Oscillatory, if it is neither finally positive nor finally negative; and

(iii) Nonoscillatory, if it is either finally positive or finally negative (Bainov and Simeonov, 1998; Isaac and Lipcsey, 2010b).

\section{The Existence of Nonoscillatory Solutions}

We return to Equation (1.1) and introduce Conditions C2.1 - C2.4:

C2.1 If $\tau_{i}>0, i \in I_{m}=\{1,2, \cdots, m\} ; \sigma_{\ell} \geq 0, \ell \in I_{m_{j}}=\left\{1,2, \cdots, m_{j}\right\}$.

C2.2 $p_{i}(t) \in P C^{1}\left(\left[t_{0}, T\right), \mathbb{R}\right) ; p_{i k} \geq 0, i \in I_{m}, k \in \mathbb{Z} ; f, g \in C\left(\left[t_{0}, T\right) \times \mathbb{R}, \mathbb{R}\right)$.

C2.3 $p_{i}(t) \geq 0, \sum_{i=1}^{m} p_{i}(t) \leq A ; \sum_{i=1}^{m} p_{i k} \geq A_{k},\left(0<A, A_{k}<1\right), k \in Z$ for all sufficiently large $t$ and there exit $p_{i}(t) \geq a_{0}>0$ and $p_{i k} \geq a_{0 k}>0$, for some $i \in I_{m}, k \in \mathbb{Z}$.

$$
\text { C2.4 }\left\{\begin{array}{l}
f\left(t, u_{1}, \cdots, u_{m_{j}}\right) \geq 0, g\left(t_{k}, u_{1}, \cdots, u_{m_{j}}\right) \geq 0 \text { if } u_{\ell} \geq 0, \ell \in I_{m_{j}} ; \\
f\left(t, u_{1}, \cdots, u_{m_{j}}\right) \geq f\left(t, v_{1}, \cdots, v_{m_{j}}\right), g\left(t_{k}, u_{1}, \cdots, u_{m_{j}}\right) \geq g\left(t_{k}, v_{1}, \cdots, v_{m_{j}}\right) \\
\text { if } u_{\ell} \geq v_{\ell} \geq 0 \text { for all } \ell \in I_{m_{j}}, k \in \mathbb{Z} .
\end{array}\right.
$$

\section{Definition 2.1}

A family of functions $\mathcal{F}$ is said to be quasi-equicontinuous in $\left[t_{0}, T\right)$ if for each $\varepsilon>0$ there exists $\mu>0$ such that if $y \in \mathcal{F}, k \in \mathbb{Z}, t^{\prime}, t^{\prime \prime} \in\left[t_{k-1}, t_{k}\right) \cap\left[t_{0}, T\right)$ and $\left|t^{\prime}-t^{\prime \prime}\right|<\mu$, then $\left|y\left(t^{\prime}\right)-y\left(t^{\prime \prime}\right)\right|<\varepsilon$ (Bainov and Simeonov, 1998).

\section{Definition 2.2}

A set $\mathcal{F} \subset P C\left(\left[t_{0}, T\right), \mathbb{R}\right)$ is relatively compact if the following conditions hold:

(i) $\mathcal{F}$ is bounded, i.e., $|y(t)| \leq M$ for all $y \in \mathcal{F}, t \in\left[t_{0}, T\right)$ and some $M>0$.

(ii) $\mathcal{F}$ is quasi-equicontinuous in $\left[t_{0}, T\right)$.

Theorem 2.1 Assume that Conditions C2.1 - C2.4 hold. Let

$$
\left|p_{i}\left(t^{\prime}\right)-p_{i}\left(t^{\prime \prime}\right)\right| \leq h_{0}\left|t^{\prime}-t^{\prime \prime}\right|,\left|p_{i}\left(t_{k}^{\prime}\right)-p_{i}\left(t_{k}^{\prime \prime}\right)\right| \leq h_{0}\left|t_{k}^{\prime}-t_{k}^{\prime \prime}\right|
$$

where $h_{0}$ is a constant and there exists another constant $h_{1}>0$ such that

$$
\left\{\begin{array}{l}
\sup _{t>t_{0}} f\left(t, \exp \left(-h_{1}\left(t-\sigma_{1}\right)\right), \cdots, \exp \left(-h_{1}\left(t-\sigma_{n}\right)\right)\right)=D<\infty \\
\sup _{t_{k}>t_{0}} g\left(t_{k}, \exp \left(-h_{1}\left(t_{k}-\sigma_{1}\right)\right), \cdots, \exp \left(-h_{1}\left(t_{k}-\sigma_{n}\right)\right)\right)=D_{k}<\infty
\end{array}\right.
$$


and

$$
\begin{aligned}
& \sum_{i=1}^{m} p_{i}(t) \exp \left(h_{1} \tau_{i}\right)+\exp \left(h_{1} t\right) \sum_{i=1}^{m} p_{i k} \exp \left(-h_{1}\left(t_{k}-\tau_{i}\right)\right)+ \\
& +\exp \left(h_{1} t\right) \int_{t}^{T} f\left(s, \exp \left(-h_{1}\left(s-\sigma_{1}\right)\right), \cdots, \exp \left(-h_{1}\left(s-\sigma_{m_{j}}\right)\right)\right) d s+ \\
& +\exp \left(h_{1} t\right) \sum_{t \leq t_{k}<T} g\left(t_{k}, \exp \left(-h_{1}\left(t_{k}-\sigma_{1}\right)\right), \cdots, \exp \left(-h_{1}\left(t_{k}-\sigma_{m_{j}}\right)\right)\right) \leq 1
\end{aligned}
$$

for all sufficiently large $t$. Then Equation (1.1) has a nonoscillatory solution which converges to zero as $t \rightarrow \infty$.

Proof. We return to the family of quasi-equicontinuous functions $\mathcal{F}$ and set

$$
\mathcal{F}=y(t) \in P C\left(\left[t_{0}, T\right), \mathbb{R}\right):\left\{\begin{array}{l}
\exp \left(-h_{2} t\right) \leq y(t) \leq \exp \left(-h_{1} t\right) \\
\left|y\left(t^{\prime}\right)-y\left(t^{\prime \prime}\right)\right| \leq L\left|t^{\prime}-t^{\prime \prime}\right| ;\left|y\left(t_{k}^{\prime}\right)-y\left(t_{k}^{\prime \prime}\right)\right| \leq L\left|t_{k}^{\prime}-t_{k}^{\prime \prime}\right|
\end{array}\right.
$$

for $t^{\prime} \leq t^{\prime \prime} \leq t_{0}$ and $\mathrm{k}: t_{k}^{\prime} \leq t_{k}^{\prime \prime} \leq t_{0}$, where $h_{2}$ is sufficiently large such that $h_{2}>h_{1}$,

$$
\begin{aligned}
& \sum_{i=1}^{m} p_{i}(t) \exp \left(h_{2} \tau_{i}\right)+\exp \left(h_{2} t\right) \sum_{i=1}^{m} p_{i k} \exp \left(h_{2}\left(t_{k}-\tau_{i}\right)\right) \geq 1, \\
& k \in \mathbb{Z} ; L \geq \max \left\{h_{0}, h_{2}\right\}
\end{aligned}
$$

and

$$
A+A_{k}+\frac{D}{L}+\frac{D_{k}}{L}<1
$$

Let us denote by $A_{B}$, all bounded piece-wise continuous functions in $P C\left(\left[t_{0}, T\right)\right)$ and define a norm in $A_{B}$ as follows:

$$
\|y\|:=\sup _{t \geq t_{0}}|y(t)| .
$$

Endowed with this norm, $A_{B}$ is a Banach space and $\mathcal{F}$ is a bounded convex closed set in $A_{B}$.

We define a mapping $\varphi$ as follows:

$$
(\varphi y)(t):=\left\{\begin{array}{l}
\sum_{i=1}^{m} p_{i}(t) y\left(t-\tau_{i}\right)+\sum_{i=1}^{m} p_{i k} y\left(t_{k}-\tau_{i}\right)+ \\
+\int_{t}^{T} f\left(s, y\left(s-\sigma_{1}\right), \cdots, y\left(s-\sigma_{m_{j}}\right)\right) d s+ \\
+\sum_{t \leq t_{k}<T} g\left(t_{k}, y\left(t_{k}-\sigma_{1}\right), \cdots, y\left(t_{k}-\sigma_{m_{j}}\right)\right), t \geq T \\
\exp \left(\frac{\ln (\varphi y)(T)}{T}\right), t_{0} \leq t<T,
\end{array}\right.
$$

where $T$ is sufficiently large. Precisely,

$$
T \geq t_{0}+\max \left\{\tau_{1}, \cdots, \tau_{m} ; \sigma_{1}, \cdots, \sigma_{m_{j}}\right\} .
$$

Clearly by virtue of the proposed value of $T$ above, Inequality (2.3) holds and

$$
\left\{\begin{array}{l}
\sum_{i=1}^{m} p_{i}\left(t^{\prime \prime}\right)+\sum_{i=1}^{m} \exp \left(-h_{1}\left(t^{\prime}-\tau_{i}\right)\right)+\frac{D}{L} \leq \frac{1}{2}, \text { for } t^{\prime \prime} \geq t^{\prime} \geq T, \\
\sum_{i=1}^{m} p_{i}\left(t_{k}^{\prime \prime}\right)+\sum_{i=1}^{m} \exp \left(-h_{1}\left(t_{k}^{\prime}-\tau_{i}\right)\right)+\frac{D_{k}}{L} \leq \frac{\left|t^{\prime \prime}-t^{\prime}\right|}{2 \mid t_{k}^{\prime \prime}-t_{k}^{\prime}}, \text { for } t_{k}^{\prime \prime} \geq t_{k}^{\prime} \geq T .
\end{array}\right.
$$

At this point, we need to prove the following facts:
(a) $\varphi \mathcal{F} \subset \mathcal{F}$;
(b) If $\lim _{j \rightarrow \infty}\left\|y_{j}-y\right\|=0$, then $y \in \mathcal{F}$, where $y_{j} \in \mathcal{F}$ is a sequence;
(c) $\varphi \mathcal{F}$ is relatively compact. 
Let us now examine their verification one after the other.

(a) For $t \geq T$ and $\mathrm{k}$ : $t_{k} \geq T$, we obtain, for $y \in \mathcal{F}$,

$$
\begin{aligned}
(\varphi y)(t) \leq & \sum_{i=1}^{m} p_{i}(t) \exp \left(-h_{1}\left(t-\tau_{i}\right)\right)+\sum_{i=1}^{m} p_{i k} \exp \left(-h_{1}\left(t_{k}-\tau_{i}\right)\right)+ \\
& +\int_{t}^{T} f\left(s, \exp \left(-h_{1}\left(s-\sigma_{1}\right)\right), \cdots, \exp \left(-h_{1}\left(s-\sigma_{m_{j}}\right)\right)\right) d s+ \\
& +\sum_{t \leq t_{k}<T} g\left(t_{k}, \exp \left(-h_{1}\left(t_{k}-\sigma_{1}\right)\right), \cdots, \exp \left(-h_{1}\left(t_{k}-\sigma_{m_{j}}\right)\right)\right) \\
= & \exp \left(-h_{1} t\right)\left[\sum_{i=1}^{m} p_{i}(t) \exp \left(h_{1} \tau_{i}\right)+\exp \left(h_{1} t\right) \sum_{i=1}^{m} p_{i k} \exp \left(-h_{1}\left(t_{k}-\tau_{i}\right)\right)+\right. \\
& +\exp \left(h_{1} t\right) \int_{t}^{T} f\left(s, \exp \left(-h_{1}\left(s-\sigma_{1}\right)\right), \cdots, \exp \left(-h_{1}\left(s-\sigma_{m_{j}}\right)\right)\right) d s+ \\
& \left.+\exp \left(h_{1} t\right) \sum_{t \leq t_{k}<T} g\left(t_{k}, \exp \left(-h_{1}\left(t_{k}-\sigma_{1}\right)\right), \cdots, \exp \left(-h_{1}\left(t_{k}-\sigma_{m_{j}}\right)\right)\right)\right] \\
\leq & \exp \left(-h_{1} t\right) .
\end{aligned}
$$

The first inequality is due to Equation (2.4) and the definition of $\mathcal{F}$ and the last inequality is because of Inequality (2.3). At the same time, using analogous reasoning, we obtain

$$
\begin{aligned}
(\varphi y)(t) & \geq \sum_{i=1}^{m} p_{i}(t) \exp \left(-h_{2}\left(t-\tau_{i}\right)\right)+\sum_{i=1}^{m} p_{i k} \exp \left(-h_{2}\left(t_{k}-\tau_{i}\right)\right) \\
& =\exp \left(-h_{2} t\right)\left[\sum_{i=1}^{m} p_{i}(t) \exp \left(h_{2} \tau_{i}\right)+\exp \left(h_{2} t\right) \sum_{i=1}^{m} p_{i k} \exp \left(-h_{2}\left(t_{k}-\tau_{i}\right)\right)\right] \\
& \geq \exp \left(-h_{2} t\right) .
\end{aligned}
$$

Consequently,

$$
\exp \left(-h_{2} T\right) \leq(\varphi y)(T) \leq \exp \left(-h_{1} T\right)
$$

which is equivalent to

$$
-h_{2} \leq \frac{\ln (\varphi y)(T)}{T} \leq-h_{1} .
$$

Expressions (2.4) and (2.6) imply that $(\varphi y)(t) \in P C\left(\left[t_{0}, T\right)\right)$ and

$$
\exp \left(-h_{2} t\right) \leq(\varphi y)(t) \leq \exp \left(-h_{1} t\right), \forall t \geq t_{0} \text { and } t_{k} \geq t_{0}
$$


For $t^{\prime \prime} \geq t^{\prime} \geq T$ and $\mathrm{k}: t_{k}^{\prime \prime} \geq t_{k}^{\prime} \geq T$, we obtain

$$
\begin{aligned}
& \left|(\varphi y)\left(t^{\prime \prime}\right)-(\varphi y)\left(t^{\prime}\right)\right| \leq \sum_{i=1}^{m} \mid p_{i}\left(t^{\prime \prime}\right) y\left(t^{\prime \prime}-\tau_{i}\right)+p_{i}\left(t_{k}^{\prime \prime}\right) y\left(t_{k}^{\prime \prime}-\tau_{i}\right)-p_{i}\left(t^{\prime}\right) y\left(t^{\prime}-\tau_{i}\right)- \\
& -p_{i}\left(t_{k}^{\prime}\right) y\left(t_{k}^{\prime}-\tau_{i}\right) \mid+\int_{t^{\prime}}^{t^{\prime \prime}} f\left(s, y\left(-h_{1}\left(s-\sigma_{1}\right)\right), \cdots, y\left(-h_{1}\left(s-\sigma_{m_{j}}\right)\right)\right) d s+ \\
& +\sum_{t^{\prime} \leq t_{k} \leq t^{\prime \prime}} g\left(t_{k}, y\left(-h_{1}\left(t_{k}-\sigma_{1}\right)\right), \cdots, y\left(-h_{1}\left(t_{k}-\sigma_{m_{j}}\right)\right)\right) \\
& \leq\left\{\sum_{i=1}^{m}\left[p_{i}\left(t^{\prime \prime}\right)+\exp \left(-h_{1}\left(t^{\prime}-\tau_{i}\right)\right)\right]\right\} L\left|t^{\prime \prime}-t^{\prime}\right|+ \\
& +\left\{\sum_{i=1}^{m}\left[p_{i}\left(t_{k}^{\prime \prime}\right)+\exp \left(-h_{1}\left(t_{k}^{\prime}-\tau_{i}\right)\right)\right]\right\} L\left|t_{k}^{\prime \prime}-t_{k}^{\prime}\right|+ \\
& +\sup _{t \geq T}\left\{f\left(s, \exp \left(-h_{1}\left(s-\sigma_{1}\right)\right), \cdots, \exp \left(-h_{1}\left(s-\sigma_{m_{j}}\right)\right)\right)\right\}\left|t^{\prime \prime}-t^{\prime}\right|+ \\
& +\sup _{t_{k} \geq T}\left\{g\left(t_{k}, \exp \left(-h_{1}\left(t_{k}-\sigma_{1}\right)\right), \cdots, \exp \left(-h_{1}\left(t_{k}-\sigma_{m_{j}}\right)\right)\right)\right\}\left|t_{k}^{\prime \prime}-t_{k}^{\prime}\right| \\
& \leq\left[\sum_{i=1}^{m} p_{i}\left(t^{\prime \prime}\right)+\sum_{i=1}^{m} \exp \left(-h_{1}\left(t^{\prime}-\tau_{i}\right)\right)+\frac{D}{L}\right] L\left|t^{\prime \prime}-t^{\prime}\right|+ \\
& +\left[\sum_{i=1}^{m} p_{i}\left(t_{k}^{\prime \prime}\right)+\sum_{i=1}^{m} \exp \left(-h_{1}\left(t_{k}^{\prime}-\tau_{i}\right)\right)+\frac{D_{k}}{L}\right] L\left|t_{k}^{\prime \prime}-t_{k}^{\prime}\right| \\
& \leq \frac{L\left|t^{\prime \prime}-t^{\prime}\right|}{2}+\frac{L\left|t^{\prime \prime}-t^{\prime}\right|}{2}=L\left|t^{\prime \prime}-t^{\prime}\right|,
\end{aligned}
$$

where the first inequality is given using the Triangle inequality. The following is based on the definition of $\mathcal{F}$ and the Mean Value Theorem. The next step is due to Equation (2.2) and the last step from Equation (2.5). Additionally, for $t_{0} \leq t^{\prime} \leq t^{\prime \prime} \leq T$, the Mean Value Theorem can be applied to Equation (2.4) leading to the result

$$
\begin{aligned}
\left|(\varphi y)\left(t^{\prime \prime}\right)-(\varphi y)\left(t^{\prime}\right)\right| & =\left|\exp \left(\frac{\ln (\varphi y)(T)}{T} t^{\prime \prime}\right)-\exp \left(\frac{\ln (\varphi y)(T)}{T} t^{\prime}\right)\right| \\
& \leq h_{2}\left|t^{\prime \prime}-t^{\prime}\right| \leq L\left|t^{\prime \prime}-t^{\prime}\right|
\end{aligned}
$$

Thus,

$$
\left|(\varphi y)\left(t^{\prime \prime}\right)-(\varphi y)\left(t^{\prime}\right)\right| \leq L\left|t^{\prime \prime}-t^{\prime}\right|
$$

for $t^{\prime \prime} \geq t^{\prime} \geq t_{0}$. Therefore, $\varphi y \in \mathcal{F}$.

(b) By definition, $\varphi$ is a piece-wise continuous mapping. Assume the existence of a sequence $y_{j} \in \mathcal{F}$ such that

$$
\lim _{j \rightarrow \infty}\left\|y_{j}(t)-y(t)\right\|=0
$$

then $y \in \mathcal{F}$. 
Indeed, for $t \geq T$ and $\mathrm{k}$ such that $t_{k} \geq T$,

$$
\begin{aligned}
& \left|\left(\varphi y_{j}\right)(t) \quad-\quad(\varphi y)(t)\right| \leq \sum_{i=1}^{m} p_{i}(t)\left|y_{j}\left(t-\tau_{i}\right)-y\left(t-\tau_{i}\right)\right|+ \\
& +\sum_{i=1}^{m} p_{i k}\left|y_{j}\left(t_{k}-\tau_{i}\right)-y\left(t_{k}-\tau_{i}\right)\right|+ \\
& +\int_{t}^{\infty} \mid f\left(s, y_{j}\left(s-\sigma_{1}\right), \cdots, y_{j}\left(s-\sigma_{m_{j}}\right)\right)- \\
& -f\left(s, y\left(s-\sigma_{1}\right), \cdots, y\left(s-\sigma_{m_{j}}\right)\right) \mid d s+ \\
& +\sum_{t \leq t_{k}<\infty} \mid g\left(t_{k}, y_{j}\left(t_{k}-\sigma_{1}\right), \cdots, y_{j}\left(t_{k}-\sigma_{m_{j}}\right)\right)- \\
& -g\left(t_{k}, y\left(t_{k}-\sigma_{1}\right), \cdots, y\left(t_{k}-\sigma_{m_{j}}\right)\right) \mid \\
& \leq \sum_{i=1}^{m} p_{i}(t) \sup _{t \geq t_{0}}\left|y_{j}\left(t-\tau_{i}\right)-y\left(t-\tau_{i}\right)\right|+ \\
& +\sum_{i=1}^{m} p_{i k}\left|y_{j}\left(t_{k}-\tau_{i}\right)-y\left(t_{k}-\tau_{i}\right)\right|+ \\
& +\int_{T}^{\infty} \mid f\left(s, y_{j}\left(s-\sigma_{1}\right), \cdots, y_{j}\left(s-\sigma_{m_{j}}\right)\right)- \\
& -f\left(s, y\left(s-\sigma_{1}\right), \cdots, y\left(s-\sigma_{m_{j}}\right)\right) \mid d s+ \\
& +\sum_{T \leq t_{k}<\infty} \mid g\left(t_{k}, y_{j}\left(t_{k}-\sigma_{1}\right), \cdots, y_{j}\left(t_{k}-\sigma_{m_{j}}\right)\right)- \\
& -g\left(t_{k}, y\left(t_{k}-\sigma_{1}\right), \cdots, y\left(t_{k}-\sigma_{m_{j}}\right)\right) \mid \\
& \leq\left\|y_{j}\left(t-\tau_{i}\right)-y\left(t-\tau_{i}\right)\right\|+\sum_{i=1}^{m} p_{i k}\left|y_{j}\left(t_{k}-\tau_{i}\right)-y\left(t_{k}-\tau_{i}\right)\right|+ \\
& +\int_{T}^{\infty} F_{j}(s) d s+\sum_{T \leq t_{k}<\infty} G_{j}\left(t_{k}\right)
\end{aligned}
$$

where

$$
F_{j}(s)=\left|f\left(s, y_{j}\left(s-\sigma_{1}\right), \cdots, y_{j}\left(s-\sigma_{m_{j}}\right)\right)-f\left(s, y\left(s-\sigma_{1}\right), \cdots, y\left(s-\sigma_{m_{j}}\right)\right)\right|
$$

and

$$
G_{j}\left(t_{k}\right)=\left|g\left(t_{k}, y_{j}\left(t_{k}-\sigma_{1}\right), \cdots, y_{j}\left(t_{k}-\sigma_{m_{j}}\right)\right)-g\left(t_{k}, y\left(t_{k}-\sigma_{1}\right), \cdots, y\left(t_{k}-\sigma_{m_{j}}\right)\right)\right| .
$$

The first inequality is obtained from Equation (2.4) and the last steps are because of the definition of a norm in $A_{B}$.

Obviously,

$$
\lim _{j \rightarrow \infty} F_{j}(s)=0 ; \lim _{j \rightarrow \infty} G_{j}\left(t_{k}\right)=0 \text { and } \lim _{j \rightarrow \infty} \sum_{i=0}^{m} p_{i k}\left|y_{j}\left(t_{k}-\tau_{i}\right)-y\left(t_{k}-\tau_{i}\right)\right|=0 .
$$

However, expresion

$$
F_{j}(s)=2 f\left(s, \exp \left(-h_{1}\left(s-\sigma_{1}\right)\right), \cdots, \exp \left(-h_{1}\left(s-\sigma_{m_{j}}\right)\right)\right) .
$$

Therefore, in view of Equation (2.7) and Lebesgues dominated convergence theorem, we can assert that

$$
\lim _{j \rightarrow \infty}\left[\sum_{i=0}^{m} p_{i k}\left|y_{j}\left(t_{k}-\tau_{i}\right)-y\left(t_{k}-\tau_{i}\right)\right|+\int_{T}^{\infty} F_{j}(s) d s+\sum_{T \leq t_{k}<\infty} G_{j}\left(t_{k}\right)\right] .
$$

Consequently

$$
\lim _{j \rightarrow \infty}\left(\sup _{t \geq T}\left|\left(\varphi y_{j}\right)(t)-(\varphi y)(t)\right|\right)=0
$$

Hence

$$
\lim _{j \rightarrow \infty}\left|\left(\varphi y_{j}\right)(T)-(\varphi y)(T)\right|=0 .
$$


Whenever $t_{0} \leq t \leq T$, the following condition holds:

$$
\begin{aligned}
\lim _{j \rightarrow \infty}\left|\left(\varphi y_{j}\right)(t)-(\varphi y)(t)\right| & =\left|\frac{\ln \left(\varphi y_{j}\right)(T)}{T}-\frac{\ln (\varphi y)(T)}{T}\right| t \\
& \leq\left|\ln \left(\varphi y_{j}\right)(T)-\ln (\varphi y)(T)\right| .
\end{aligned}
$$

The combination of Equations (2.9) and (2.10) gives

$$
\lim _{j \rightarrow \infty}\left(\sup _{t_{0} \leq t \leq T}\left|\left(\varphi y_{j}\right)(t)-(\varphi y)(t)\right|\right)=0 .
$$

Therefore from Equations (2.8) and (2.11), it follows that

$$
\lim _{j \rightarrow \infty}\left\|\varphi y_{j}-\varphi y\right\|=0
$$

which implies $y \in \mathcal{F}$.

(c) In this final stage, we show that $\varphi \mathcal{F}$ is relatively compact. Obviously from the proofs of (a) and (b) above, $\varphi \mathcal{F}$ is uniformly bounded and quasi-equicontinuous in $\left[t_{0}, T\right)$. This implies that for each $y \in \mathcal{F}$,

$$
|(\varphi y)(t)| \leq b_{0}
$$

where $b_{0}>0$ and

$$
\left|(\varphi y)\left(t^{\prime \prime}\right)-(\varphi y)\left(t^{\prime}\right)\right| \leq L\left|t^{\prime \prime}-t^{\prime}\right|
$$

for $t^{\prime \prime} \geq t^{\prime} \geq t_{0}$ and $\mathrm{k}: t_{k}^{\prime \prime} \geq t_{k}^{\prime} \geq t_{0}$. Without loss of generality, we set

$$
b_{0}=\exp \left(-h_{1} t\right), t \geq t_{0} .
$$

Hence, for any arbitrarily pre-assigned small positive number $\varepsilon$, there exists a sufficiently large $T^{\prime}>t_{0}$ such that whenever $\exp \left(-h_{1} t\right)<\frac{\varepsilon}{2}$

$$
\left|(\varphi y)\left(t^{\prime \prime}\right)-(\varphi y)\left(t^{\prime}\right)\right| \leq \varepsilon \text { for } t, t_{k} \geq T^{\prime}, t^{\prime \prime} \geq t^{\prime} \geq T^{\prime} \text { and } k: t_{k}^{\prime \prime} \geq t_{k}^{\prime} \geq T^{\prime} .
$$

On the other hand, if we set $\delta=\frac{\varepsilon}{L}$ and assume that $\left|t^{\prime \prime}-t^{\prime}\right|<\delta$, then for all $t_{0} \leq t^{\prime} \leq t^{\prime \prime} \leq T^{\prime}$ and $\mathrm{k}: t_{0} \leq t_{k}^{\prime} \leq t_{k}^{\prime \prime} \leq T^{\prime}$ it becomes clear that

$$
\left|(\varphi y)\left(t^{\prime \prime}\right)-(\varphi y)\left(t^{\prime}\right)\right| \leq \varepsilon
$$

Thus, from Conditions (2.12) and (2.13), we can affirm that $\varphi \mathcal{F}$ is quasi-equicontinuous in $\left[t_{0}, T\right.$ ) and hence, $\varphi \mathcal{F}$ is relatively compact. By virtue of Schauder Tikhonov Fixed Point Theorem, the mapping $\varphi$ has a fixed point $y^{*}(t) \in \mathcal{F}$ which is a nonoscillatory solution of Equation (1.1) and converges to zero when $t \rightarrow \infty$. This completes the proof of Theorem 2.1.

Corollary 2.1 Assuming that the function $p_{i}(t)$ satisfies Conditions $\mathrm{C} 2.2$ and $\mathrm{C} 2.3$ as well as $q_{j}(t) \in P C\left(R_{+}, R_{+}\right)$and $q_{j k} \geq 0$, the following two conditions hold. If $p_{i}(t) \leq p_{i}, q_{j}(t) \leq q_{j}$ and there exists a positive $\lambda$ such that

$$
\begin{aligned}
\sum_{i=1}^{m} p_{i} \exp \left(\lambda \tau_{i}\right) & +\exp (\lambda t) \sum_{i=1}^{m} p_{i k} \exp \left(-\lambda\left(t_{k}-\tau_{i}\right)\right)+ \\
& +\sum_{j=1}^{n} q_{j} \exp \left(\lambda \sigma_{j}\right)\left[\frac{1}{\lambda}+\sum_{t \leq t_{k}<\infty} \exp \left(-\lambda t_{k}\right)\right] \leq 1
\end{aligned}
$$

then equation

$$
\left\{\begin{array}{l}
{\left[y(t)-\sum_{i=1}^{m} p_{i}(t) y\left(t-\tau_{i}\right)\right]^{\prime}+\sum_{j=1}^{n} q_{j}(t) y\left(t-\sigma_{j}\right)=0, \quad t \notin S} \\
\Delta\left[y\left(t_{k}\right)-\sum_{i=1}^{m} p_{i k} y\left(t_{k}-\tau_{i}\right)\right]+\sum_{j=1}^{n} q_{j k} y\left(t_{k}-\sigma_{j}\right)=0, \quad \forall t_{k} \in S
\end{array}\right.
$$

has a nonoscillatory solution which converges to zero as $t \rightarrow \infty$.

Remark 2.1 When $p_{i}(t) \equiv p_{i}$ and $q_{j}(t) \equiv q_{j}$, Inequality (2.14) is equivalent to the characteristic system of Equation (2.15) which has no solutions in $R_{+} \times[0,1)$. Therefore, Inequality (2.14) is a necessary and sufficient condition for Equation (2.15) with constant coefficients to have a nonoscillatory solution (Bainov and Simeonov, 1998). 
Corollary 2.2 Consider the equation

$$
\begin{cases}\left.\left[y(t)-\sum_{i=1}^{m} p_{i}(t) y\left(t-\tau_{i}\right)\right]^{\prime}+\left.\sum_{j=1}^{n} q_{j}(t)\left[\prod_{\ell=1}^{m_{j}} \mid y(t)-\sigma_{j \ell}\right)\right|^{\alpha_{j \ell}}\right] \operatorname{sign}(y(t))=0 & t \notin S \\ \left.\Delta\left[y\left(t_{k}\right)-\sum_{i=1}^{m} p_{i k} y\left(t_{k}-\tau_{i}\right)\right]+\left.\sum_{j=1}^{n} q_{j k}\left[\prod_{\ell=1}^{m_{j}} \mid y\left(t_{k}\right)-\sigma_{j \ell}\right)\right|^{\alpha_{j \ell}}\right] \operatorname{sign}\left(y\left(t_{k}\right)\right)=0, & \forall t_{k} \in S\end{cases}
$$

for $t \geq t_{0}>0$ and $k: t_{k} \geq t_{0}>0$. In Equation (2.16), it is assumed that $\tau_{i}>0, \sigma_{j \ell} \geq 0\left(i \in I_{m}, j \in I_{n}\right.$ and $\ell \in I_{m_{j}}=$ $\left.\left\{1,2, \cdots, m_{j}\right\}\right) ; p_{i}(t)$ satisfies Conditions $\mathrm{C} 2.2$ and $\mathrm{C} 2.3 ; q_{j}(t) \in P C\left(R_{+}, R_{+}\right)$and $q_{j k} \geq 0$. If there exist a small positive number $\lambda$ such that for some sufficiently large $T$,

$$
\left\{\begin{array}{l}
\sup _{t \geq T}\left[q_{j}(t) \exp \left(-\lambda \sum_{\ell=1}^{m_{j}} \alpha_{j \ell} t\right)\right]<\infty, \forall j \in I_{n}, t \notin S \\
\sup _{t_{k} \geq T}\left[q_{j k} \exp \left(-\lambda_{k} \sum_{\ell=1}^{m_{j}} \alpha_{j \ell} t_{k}\right)\right]<\infty, \forall j \in I_{n}, \forall t_{k} \in S
\end{array}\right.
$$

and

$$
\left\{\begin{array}{l}
\sup _{t \geq T}\left\{p_{i}(t) \exp \left(-\lambda \tau_{i}\right)+\sum_{j=1}^{n} \exp \left(\lambda \sum_{\ell=1}^{m_{j}} \alpha_{j \ell} \sigma_{j \ell}\right) *\right. \\
\left.* \int_{t}^{\infty} q_{j}(s) \exp \left[-\lambda\left(\sum_{\ell=1}^{m_{j}} \alpha_{j \ell} s-s\right)\right] d s\right\} \leq 1, t \notin S \\
\sup _{t_{k} \geq T}\left\{p_{i k} \exp \left(-\lambda_{k} \tau_{i}\right)+\sum_{j=1}^{n} \exp \left(\lambda_{k} \sum_{\ell=1}^{m_{j}} \alpha_{j \ell} \sigma_{j \ell}\right) *\right. \\
\left.* \sum_{t \leq t_{k}<\infty} q_{j k} \exp \left[-\lambda_{k}\left(\sum_{\ell=1}^{m_{j}} \alpha_{j \ell} t_{k}-t_{k}\right)\right] d s\right\} \leq 1, \forall t_{k} \in S
\end{array}\right.
$$

then Equation (2.16) has a nonoscillatory solution which converges to zero as $t \rightarrow \infty$.

\section{Oscillatory Conditions}

We now consider the nonlinear neutral delay impulsive differential equation with variable coefficients

$$
\begin{cases}\left.\left[y(t)-\sum_{i=1}^{m} p_{i}(t) y\left(t-\tau_{i}\right)\right]^{\prime}+\left.q(t)\left[\prod_{\ell=1}^{m_{j}} \mid y(t)-\sigma_{\ell}\right)\right|^{\alpha_{\ell}}\right] \operatorname{sign}(y(t))=0 & t \notin S \\ \left.\Delta\left[y\left(t_{k}\right)-\sum_{i=1}^{m} p_{i k} y\left(t_{k}-\tau_{i}\right)\right]+\left.q_{k}\left[\prod_{\ell=1}^{m_{j}} \mid y\left(t_{k}\right)-\sigma_{\ell}\right)\right|^{\alpha_{\ell}}\right] \operatorname{sign}\left(y\left(t_{k}\right)\right)=0, & \forall t_{k} \in S\end{cases}
$$

for $t \geq t_{0}>0$ and $\forall k: t_{k} \geq t_{0}>0$. We introduce Conditions C3.1 to C3.4:

C3.1 $0<\tau_{1} \leq \tau_{2} \leq \cdots \leq \tau_{m}, \lim _{t \rightarrow+\infty}\left(t-\tau_{i}\right)=+\infty$;

C3.2 $0<\sigma_{1} \leq \sigma_{2} \leq \cdots \leq \sigma_{m_{j}}, \lim _{t \rightarrow+\infty}\left(t-\sigma_{\ell}\right)=+\infty ; \alpha_{\ell} \geq 0$ and $\sum_{\ell=1}^{m_{j}} \alpha_{\ell}=1$;

C3.3 $p_{i}(t) \in P C^{1}\left(R_{+}, R_{+}\right)$and $p_{i k} \in \mathbb{R}, k \in \mathbb{Z}$;

C3.4 $q_{j}(t) \in P C\left(R_{+}, R_{+}\right)$and $q_{j k} \geq 0, k \in \mathbb{Z}$.

Denote $\zeta=\max _{1 \leq i \leq m ; 1 \leq \ell \leq m_{j}}\left\{\tau_{i}, \sigma_{\ell}\right\}$. If $m_{j}=1$, Equation (3.1) can be reduced to

$$
\begin{cases}{\left[y(t)-\sum_{i=1}^{m} p_{i}(t) y\left(t-\tau_{i}\right)\right]^{\prime}+q(t) y(t-\sigma)=0} & t \notin S \\ \Delta\left[y\left(t_{k}\right)-\sum_{i=1}^{m} p_{i k} y\left(t_{k}-\tau_{i}\right)\right]+q_{k} y\left(t_{k}-\sigma\right)=0, & \forall t_{k} \in S\end{cases}
$$

Next, we establish the following lemmas which will be useful in the proof of the main result.

Lemma 3.1 Assume that Condition C3.4 is satisfied with $\sum_{i=1}^{m} p_{i}(t)$ bounded and non-negative, and there exists $t^{*} \geq t_{0}$ such that

$$
\sum_{i=1}^{m} p_{i}\left(t^{*}+n \tau_{i}\right) \leq 1, n=0,1, \cdots
$$


Let $y(t)$ be a finally positive solution of Equation (3.1). The function $z(t)$ is defined as follows

$$
z(t)=y(t)-\sum_{i=1}^{m} p_{i}(t) y\left(t-\tau_{i}\right) .
$$

Then finally $z(t)>0$ with $z^{\prime}(t)<0$ and $\Delta z\left(t_{k}\right)<0$.

Proof. From Equation (3.1) we can affirm that $z^{\prime}(t)<0$ and $\Delta z\left(t_{k}\right)<0$ finally. It remains to show that $z(t)>0$ finally. By contradiction, we assume that $z(t)$ is finally negative. This implies that there exist a sufficiently large $T$ such that $z(t)<-d<0$ for all $t \geq T$, where $d$ is a positive constant. Hence

$$
y(t) \leq-d+\sum_{i=1}^{m} p_{i}(t) y\left(t-\tau_{i}\right), \text { for all } t \geq T .
$$

In particular,

$$
y\left(t^{*}+(n+N) \tau_{i}\right) \leq-n d+y\left(t^{*}+(N-1) \tau_{i}\right), i \in I_{m}, n=1,2, \cdots .
$$

if $t^{*}+N \tau_{i} \geq T$. Hence, $y(t)$ cannot be finally positive. This contradicts the initial assumption of the Lemma and hence completes the proof.

Lemma 3.2 Assume that Conditions C3.2 and C3.4 with the inequalities

$$
\left\{\begin{array}{l}
\lim _{t \rightarrow \infty} \inf \frac{y(t)}{y\left(t-\sigma_{\ell}\right)} \leq r \leq 1 \\
\lim _{t_{k} \rightarrow \infty} \inf \frac{y\left(t_{k}\right)}{y\left(t_{k}-\sigma_{\ell}\right)} \leq r_{k} \leq 1
\end{array}\right.
$$

are fulfilled. Further, let us suppose that

$$
\left\{\begin{array}{l}
\liminf _{t \rightarrow \infty} \int_{t-\sigma_{\ell}}^{t} q(s) d s>0 \\
\lim _{t_{k} \rightarrow \infty} \inf \int_{t_{k}-\sigma_{\ell}}^{t_{k}} q(s) d s>0
\end{array}\right.
$$

and the solution of Equation (3.1) be such that the solution $\left(\lambda(t), \lambda_{k}\right)$ of the associated generalized characteristic system satisfies the inequalities

$$
\left\{\begin{array}{l}
\lambda(t) \geq q(t) \exp \left(\int_{t-\sigma_{\ell}}^{t} \lambda(s) d s\right) \prod_{t-\sigma_{\ell} \leq t_{j}<t}\left(1-\lambda_{j}\right)^{-1}, t \geq t_{0} \\
\lambda_{k} \geq q_{k} \exp \left(\int_{t_{k}-\sigma_{\ell}}^{t_{k}} \lambda(s) d s\right) \prod_{t_{k}-\sigma_{\ell} \leq t_{j}<t_{k}}\left(1-\lambda_{j}\right)^{-1}, t_{k} \geq t_{0} .
\end{array}\right.
$$

Then

$$
\left\{\begin{array}{l}
\lim _{t \rightarrow \infty} \inf \left\{\left(\int_{t-\sigma_{\ell}}^{t} \lambda(s) d s\right) \prod_{t-\sigma_{\ell} \leq t_{j}<t}\left(1-\lambda_{j}\right)^{-1}\right\}<\infty \\
\lim _{t_{k} \rightarrow \infty} \inf \left\{\left(\int_{t_{k}-\sigma_{\ell}}^{t_{k}} \lambda(s) d s\right)_{t_{k}-\sigma_{\ell} \leq t_{j}<t_{k}}\left(1-\lambda_{j}\right)^{-1}\right\}<\infty
\end{array}\right.
$$

Proof. If we define

$$
\left\{\begin{array}{l}
Q(t):=\int_{t_{0}}^{t} q(s) d s, t \geq t_{0} \\
Q\left(t_{k}\right):=\int_{t_{0}}^{t_{k}} q(s) d s, t_{k} \geq t_{0}
\end{array}\right.
$$

then Inequality (3.6) implies that

$$
\lim _{t \rightarrow \infty} Q(t)=+\infty, \lim _{t_{k} \rightarrow \infty} Q\left(t_{k}\right)=+\infty
$$

and $Q(t), Q\left(t_{k}\right)$ are strictly increasing. Then $Q^{-1}(t)$ and $Q^{-1}\left(t_{k}\right)$ are well defined, strictly increasing and

$$
\lim _{t \rightarrow \infty} Q^{-1}(t)=+\infty, \lim _{t_{k} \rightarrow \infty} Q^{-1}\left(t_{k}\right)=+\infty
$$

Indeed, Inequality (3.6) means there exist $b, b_{k}>0$ and $T \geq t_{0}$ such that 


$$
\left\{\begin{array}{l}
Q(t)-Q\left(t-\sigma_{\ell}\right) \geq \frac{b}{2}, \forall t \geq T \\
Q\left(t_{k}\right)-Q\left(t_{k}-\sigma_{\ell}\right) \geq \frac{b_{k}}{2}, \forall t_{k} \geq T
\end{array}\right.
$$

and thus,

$$
\left\{\begin{array}{l}
Q^{-1}\left(Q(t)-\frac{b}{2}\right) \geq t-\sigma_{\ell}, \forall t \geq T \\
Q^{-1}\left(Q\left(t_{k}\right)-\frac{b_{k}}{2}\right) \geq t_{k}-\sigma_{\ell}, \forall t_{k} \geq T .
\end{array}\right.
$$

Now set

$$
\left\{\begin{array}{l}
\Lambda(t)=\exp \left(-\int_{T}^{t} \lambda(s) d s\right) \prod_{T \leq t_{j}<t}\left(1-\lambda_{j}\right) \\
\Lambda_{k}=\exp \left(-\int_{T}^{t_{k}} \lambda(s) d s\right) \prod_{T \leq t_{j}<t_{k}}\left(1-\lambda_{j}\right) .
\end{array}\right.
$$

Inequality (3.7) involves

$$
\left\{\begin{array}{l}
\Lambda^{\prime}(t) \leq-q(t) \Lambda\left(t-\sigma_{\ell}\right), \forall t \geq T \\
\Delta \Lambda\left(t_{k}\right) \leq-q_{k} \Lambda\left(t_{k}-\sigma_{\ell}\right), \forall t_{k} \geq T .
\end{array}\right.
$$

By virtue of Inequality (3.5), the ratios $\frac{\Lambda\left(t-\sigma_{\ell}\right)}{\Lambda(t)}$ and $\frac{\Lambda\left(t_{k}-\sigma_{\ell}\right)}{\Lambda\left(t_{k}\right)}$ are bounded above under Inequality (3.6). This implies that Inequality (3.8) is valid and completes the proof.

Let us now prove the following result.

Theorem 3.1 Let Conditions C3.1 - C3.4 be fulfilled. In addition, let us assume that Inequality (3.6) is satisfied, and either

$$
\left\{\begin{array}{c}
\lim _{t \rightarrow \infty} \inf \left\{\operatorname { i n f } _ { \lambda > 0 } \left[\prod_{\ell=1}^{m_{j}} \sum_{i=1}^{m} p_{i}^{\alpha_{\ell}}\left(t-\sigma_{\ell}\right) \frac{q(t)}{q\left(t-\tau_{i}\right)} \exp \left(\lambda \tau_{i}\right)(1-\mu)^{-\tau_{i}}+\right.\right. \\
\left.\left.+\frac{q(t)}{\lambda} \exp \left(\lambda \sum_{\ell=1}^{m_{j}} \alpha_{\ell} \sigma_{\ell}\right)(1-\mu)^{-\sigma_{\ell}}\right]\right\}>1 \\
\lim _{t_{k} \rightarrow \infty} \inf \left\{\operatorname { i n f } _ { \lambda _ { k } > 0 } \left[\prod_{\ell=1}^{m_{j}} \sum_{i=1}^{m} p_{i}^{\alpha_{\ell}}\left(t_{k}-\sigma_{\ell}\right) \frac{q_{k}}{q\left(t_{k}-\tau_{i}\right)} \exp \left(\lambda_{k} \tau_{i}\right)(1-\mu)^{-\tau_{i}}+\right.\right. \\
\left.\left.+\frac{q_{k}}{\lambda_{k}} \exp \left(\lambda_{k} \sum_{\ell=1}^{m_{j}} \alpha_{\ell} \sigma_{\ell}\right)(1-\mu)^{-\sigma_{\ell}}\right]\right\}>1
\end{array}\right.
$$

or

$$
\left\{\begin{array}{c}
\liminf _{t \rightarrow \infty}\left\{\operatorname { i n f } _ { \lambda > 0 } \left[\prod_{\ell=1}^{m_{j}} \sum_{i=1}^{m} p_{i}^{\alpha_{\ell}}\left(t-\sigma_{\ell}\right) \exp \left(\lambda \int_{t-\tau_{i}}^{t} q(s) d s\right)(1-\gamma)^{-\tau_{i}}+\right.\right. \\
\left.\left.+\frac{1}{\lambda} \exp \left(\lambda \sum_{\ell=1}^{m_{j}} \alpha_{\ell} \int_{t-\sigma_{\ell}}^{t} q(s) d s\right)(1-\gamma)^{-\sigma_{\ell}}\right]\right\}>1 \\
\lim _{t_{k} \rightarrow \infty} \inf \left\{\operatorname { i n f } _ { \lambda _ { k } > 0 } \left[\prod_{\ell=1}^{m_{j}} \sum_{i=1}^{m} p_{i}^{\alpha_{\ell}}\left(t_{k}-\sigma_{\ell}\right) \exp \left(\lambda_{k} \int_{t_{k}-\tau_{i}}^{t_{k}} q(s) d s\right)(1-\gamma)^{-\tau_{i}}+\right.\right. \\
\left.\left.+\frac{1}{\lambda_{k}} \exp \left(\lambda_{k} \sum_{\ell=1}^{m_{j}} \alpha_{\ell} \int_{t_{k}-\sigma_{\ell}}^{t_{k}} q(s) d s\right)(1-\gamma)^{-\sigma_{\ell}}\right]\right\}>1 .
\end{array}\right.
$$

Then every solution of Equation (3.1) is oscillatory.

Proof. We first assume that Condition (3.11) is satisfied. Without loss of generality, assume that Equation (3.1) has a finally positive solution $y(t)$. Let $y(t)>0, y(t-\zeta)>0$, for $t \geq T_{1} \geq t_{0}$. Then, by Lemma $3.1, z(t)>0, z^{\prime}<0$ and $\Delta z\left(t_{k}\right)<0$ for $t \geq T_{1}$ and $\forall k: t_{k} \geq T_{1}$, where $z(t)$ is defined by Equation (3.4). For $t \geq T_{1}, t \neq t_{k}$ and from Equation (3.1), we have

$$
\begin{aligned}
z^{\prime}(t) & =-q(t) \prod_{\ell=1}^{m_{j}} y^{\alpha_{\ell}}\left(t-\sigma_{\ell}\right) \\
& =-q(t) \prod_{\ell=1}^{m_{j}}\left[z\left(t-\sigma_{\ell}\right)+\sum_{i=1}^{m} p_{i}\left(t-\sigma_{\ell}\right) y\left(t-\sigma_{\ell}-\tau_{i}\right)\right]^{\alpha_{\ell}} \\
& \leq-q(t)\left[\prod_{\ell=1}^{m_{j}} z^{\alpha_{\ell}}\left(t-\sigma_{\ell}\right)+\prod_{\ell=1}^{m_{j}} \sum_{i=1}^{m} p_{i}^{\alpha_{\ell}}\left(t-\sigma_{\ell}\right) \prod_{\ell=1}^{m_{j}} y^{\alpha_{\ell}}\left(t-\sigma_{\ell}-\tau_{i}\right)\right] \\
& =-q(t) \prod_{\ell=1}^{m_{j}} z^{\alpha_{\ell}}\left(t-\sigma_{\ell}\right)+\frac{q(t)}{q\left(t-\tau_{i}\right)} \prod_{\ell=1}^{m_{j}} \sum_{i=1}^{m} p_{i}^{\alpha_{\ell}}\left(t-\sigma_{\ell}\right) z^{\prime}\left(t-\tau_{i}\right) .
\end{aligned}
$$


Notice that the first equation is due to the definition of $z(t)$ in Equation (3.4). The following inequality represents an upper estimate of the expansion on the left side and the last equation is based on Equation (3.1). Using analogous reasoning, we obtain the following result fot the corresponding impulsive part:

$$
\begin{aligned}
\Delta z\left(t_{k}\right) & =-q_{k} \prod_{\ell=1}^{m_{j}} y^{\alpha_{\ell}}\left(t_{k}-\sigma_{\ell}\right) \\
& =-q_{k} \prod_{\ell=1}^{m_{j}}\left[z\left(t_{k}-\sigma_{\ell}\right)+\sum_{i=1}^{m} p_{i}\left(t_{k}-\sigma_{\ell}\right) y\left(t_{k}-\sigma_{\ell}-\tau_{i}\right)\right]^{\alpha_{\ell}} \\
& \leq-q_{k}\left[\prod_{\ell=1}^{m_{j}} z^{\alpha_{\ell}}\left(t_{k}-\sigma_{\ell}\right)+\prod_{\ell=1}^{m_{j}} \sum_{i=1}^{m} p_{i}^{\alpha_{\ell}}\left(t_{k}-\sigma_{\ell}\right) \prod_{\ell=1}^{m_{j}} y^{\alpha_{\ell}}\left(t_{k}-\sigma_{\ell}-\tau_{i}\right)\right] \\
& =-q_{k} \prod_{\ell=1}^{m_{j}} z^{\alpha_{\ell}}\left(t_{k}-\sigma_{\ell}\right)+\frac{q_{k}}{q\left(t_{k}-\tau_{i}\right)} \prod_{\ell=1}^{m_{j}} \sum_{i=1}^{m} p_{i}^{\alpha_{\ell}}\left(t_{k}-\sigma_{\ell}\right) \Delta z\left(t_{k}-\tau_{i}\right)
\end{aligned}
$$

for all k: $t_{k} \geq T_{1}$. Set $\lambda(t)=-\frac{z^{\prime}(t)}{z(t)}$ and $\lambda_{k}=-\frac{\Delta z\left(t_{k}\right)}{z\left(t_{k}\right)}$ for each $t \geq T_{1}$ and $\mathrm{k}: t_{k} \geq T_{1}$. Therefore taking Inequality (3.7) into account, Equation (3.13) is reduced to

$$
\begin{gathered}
\lambda(t) \geq \lambda\left(t-\tau_{i}\right) \frac{q(t)}{q\left(t-\tau_{i}\right)} \prod_{\ell=1}^{m_{j}} \sum_{i=1}^{m} p_{i}^{\alpha_{\ell}}\left(t-\sigma_{\ell}\right) \exp \left(\int_{t-\tau_{i}}^{t} \lambda(s) d s\right) * \\
* \prod_{t-\tau_{i} \leq t_{j}<t}\left(1-\lambda_{j}\right)^{-1}+q(t) \exp \left(\sum_{\ell=1}^{m_{j}} \alpha_{\ell} \int_{t-\sigma_{\ell}}^{t} \lambda(s) d s\right)_{t-\sigma_{\ell} \leq t_{j}<t}\left(1-\lambda_{j}\right)^{-1}
\end{gathered}
$$

and

$$
\begin{gathered}
\lambda_{k} \geq \lambda\left(t_{k}-\tau_{i}\right) \frac{q_{k}}{q\left(t_{k}-\tau_{i}\right)} \prod_{\ell=1}^{m_{j}} \sum_{i=1}^{m} p_{i}^{\alpha_{\ell}}\left(t_{k}-\sigma_{\ell}\right) \exp \left(\int_{t_{k}-\tau_{i}}^{t_{k}} \lambda(s) d s\right) * \\
* \prod_{t_{k}-\tau_{i} \leq t_{j}<t_{k}}\left(1-\lambda_{j}\right)^{-1}+q_{k} \exp \left(\sum_{\ell=1}^{m_{j}} \alpha_{\ell} \int_{t_{k}-\sigma_{\ell}}^{t_{k}} \lambda(s) d s\right) \underset{t_{k}-\sigma_{\ell} \leq t_{j}<t_{k}}{\prod_{k}}\left(1-\lambda_{j}\right)^{-1} .
\end{gathered}
$$

It is obvious that $\lambda(t)>0$ and $\lambda_{k}>0$ for each $t \geq T_{1}$ and for all $\mathrm{k}: t_{k} \geq T_{1}$. From Inequality (3.14), we have

$$
\left\{\begin{array}{l}
\lambda(t) \geq q(t) \exp \left(\bar{\alpha} \int_{t-\sigma^{*}}^{t} \lambda(s) d s\right) \prod_{t-\sigma^{*} \leq t_{j}<t}\left(1-\lambda_{j}\right)^{-1} \\
\lambda_{k} \geq q_{k} \exp \left(\bar{\alpha} \int_{t_{k}-\sigma^{*}}^{t_{k}} \lambda(s) d s\right) \prod_{t_{k}-\sigma^{*} \leq t_{j}<t_{k}}\left(1-\lambda_{j}\right)^{-1},
\end{array}\right.
$$

where $\sigma^{*}=\min _{1 \leq \ell \leq m_{j}}\left\{\sigma_{\ell}\right\}$ and $\bar{\alpha}=\min _{1 \leq \ell \leq m_{j}}\left\{\alpha_{\ell}\right\}$. In view of Lemma 3.2, we have

$$
\left\{\begin{array}{l}
\lim _{t \rightarrow \infty} \inf \left\{\left(\int_{-\sigma^{*}}^{t} \lambda(s) d s\right) \prod_{t-\sigma^{*} \leq t_{j}<t}\left(1-\lambda_{j}\right)^{-1}\right\}<\infty \\
\lim _{t_{k} \rightarrow \infty} \inf \left\{\left(\int_{t_{k}-\sigma^{*}}^{t_{k}} \lambda(s) d s\right)_{t_{k}-\sigma^{*} \leq t_{j}<t_{k}}\left(1-\lambda_{j}\right)^{-1}\right\}<\infty .
\end{array}\right.
$$

which implies that $\lim _{t \rightarrow \infty} \inf \lambda(t)<\infty$ and $\lim _{t_{k} \rightarrow \infty} \inf \lambda_{k}<\infty$. Now we show that $\lim _{t \rightarrow \infty} \inf \lambda(t)>0$ and $\lim _{t_{k} \rightarrow \infty} \inf \lambda_{k}>0$. By contradiction, if

$$
\left\{\begin{array}{l}
\lim _{t \rightarrow \infty} \inf \lambda(t)=0 \\
\lim _{t_{k} \rightarrow \infty} \inf \lambda_{k}=0
\end{array}\right.
$$

then there would exist sequences $\left\{t_{n}\right\}$ and $\left\{t_{n k}\right\}$ such that $t_{n}, t_{n k} \geq T_{1}, \lim _{n \rightarrow \infty} t_{n}=\infty$ and $\lim _{n \rightarrow \infty} t_{n k}=\infty$ for all $k \in \mathbb{N}$. What is more, $\lambda\left(t_{n}\right) \leq \lambda(t)$ and $\lambda\left(t_{n k}\right) \leq \lambda_{k}$ for $t \in\left[T_{1}, t_{n}\right]$ and $t_{k} \in\left[T_{1}, t_{n k}\right]$ respectively. Using Inequality (3.14) again, we obtain

$$
\left\{\begin{array}{c}
\lambda\left(t_{n}\right) \geq \lambda\left(t_{n}\right) \frac{q\left(t_{n}\right)}{q\left(t_{n}-\tau_{i}\right)} \prod_{\ell=1}^{m_{j}} \sum_{i=1}^{m} p_{i}^{\alpha_{\ell}}\left(t_{n}-\sigma_{\ell}\right) \exp \left(\lambda\left(t_{n}\right) \tau_{i}\right)\left(1-\mu_{j}\right)^{-\tau_{i}}+ \\
+q\left(t_{n}\right) \exp \left(\lambda\left(t_{n}\right)\right) \sum_{\ell=1}^{m_{j}} \alpha_{\ell} \sigma_{\ell}\left(1-\mu_{j}\right)^{-\sigma_{\ell}} \\
\lambda\left(t_{n k}\right) \geq \lambda\left(t_{n k}\right) \frac{q\left(t_{n k}\right)}{q\left(t_{n k}-\tau_{i}\right)} \prod_{\ell=1}^{m_{j}} \sum_{i=1}^{m} p_{i}^{\alpha_{\ell}}\left(t_{n k}-\sigma_{\ell}\right) \exp \left(\lambda\left(t_{n k}\right) \tau_{i}\right)\left(1-\mu_{j}\right)^{-\tau_{i}}+ \\
+q\left(t_{n k}\right) \exp \left(\lambda\left(t_{n k}\right)\right) \sum_{\ell=1}^{m_{j}} \alpha_{\ell} \sigma_{\ell}\left(1-\mu_{j}\right)^{-\sigma_{\ell}} .
\end{array}\right.
$$


Hence

$$
\left\{\begin{array}{c}
\frac{q\left(t_{n}\right)}{q\left(t_{n}-\tau_{i}\right)} \prod_{\ell=1}^{m_{j}} \sum_{i=1}^{m} p_{i}^{\alpha_{\ell}}\left(t_{n}-\sigma_{\ell}\right) \exp \left(\lambda\left(t_{n}\right) \tau_{i}\right)\left(1-\mu_{j}\right)^{-\tau_{i}}+ \\
+q\left(t_{n}\right) \exp \left(\lambda\left(t_{n}\right)\right) \sum_{\ell=1}^{m_{j}} \alpha_{\ell} \sigma_{\ell}\left(1-\mu_{j}\right)^{-\sigma_{\ell}} \leq 1 \\
\frac{q\left(t_{n k}\right)}{q\left(t_{n k}-\tau_{i}\right)} \prod_{\ell=1}^{m_{j}} \sum_{i=1}^{m} p_{i}^{\alpha_{\ell}}\left(t_{n k}-\sigma_{\ell}\right) \exp \left(\lambda\left(t_{n k}\right) \tau_{i}\right)\left(1-\mu_{j}\right)^{-\tau_{i}}+ \\
+q\left(t_{n k}\right) \exp \left(\lambda\left(t_{n k}\right)\right) \sum_{\ell=1}^{m_{j}} \alpha_{\ell} \sigma_{\ell}\left(1-\mu_{j}\right)^{-\sigma_{\ell}} \leq 1
\end{array}\right.
$$

which contradicts Inequality (3.11). Therefore,

$$
\left\{\begin{array}{l}
0<\lim _{t \rightarrow \infty} \inf \lambda(t)=h<\infty \\
0<\lim _{t_{k} \rightarrow \infty} \inf \lambda\left(t_{k}\right)=h_{k}<\infty .
\end{array}\right.
$$

From Inequality (3.11), there exists $\delta \in(0,1)$ such that

$$
\left\{\begin{array}{c}
\delta \liminf _{t \rightarrow \infty}\left\{\operatorname { i n f } _ { \lambda > 0 } \left[\prod_{\ell=1}^{m_{j}} \sum_{i=1}^{m} p_{i}^{\alpha_{\ell}}\left(t-\sigma_{\ell}\right) \frac{q(t)}{q\left(t-\tau_{i}\right)} \exp \left(\lambda \tau_{i}\right)(1-\mu)^{-\tau_{i}}+\right.\right. \\
\left.\left.+\frac{q(t)}{\lambda} \exp \left(\lambda \sum_{\ell=1}^{m_{j}} \alpha_{\ell} \sigma_{\ell}\right)(1-\mu)^{-\sigma_{\ell}}\right]\right\} \geq 1 \\
\delta \lim _{t_{k} \rightarrow \infty} \inf \left\{\operatorname { i n f } _ { \lambda _ { k } > 0 } \left[\prod_{\ell=1}^{m_{j}} \sum_{i=1}^{m} p_{i}^{\alpha_{\ell}}\left(t_{k}-\sigma_{\ell}\right) \frac{q_{k}}{q\left(t_{k}-\tau_{i}\right)} \exp \left(\lambda_{k} \tau_{i}\right)(1-\mu)^{-\tau_{i}}+\right.\right. \\
\left.\left.+\frac{q_{k}}{\lambda_{k}} \exp \left(\lambda_{k} \sum_{\ell=1}^{m_{j}} \alpha_{\ell} \sigma_{\ell}\right)(1-\mu)^{-\sigma_{\ell}}\right]\right\} \geq 1
\end{array}\right.
$$

By virtue of Equation (3.15), we have

$$
\left\{\begin{array}{l}
\lambda(t)>\delta h, t \geq T_{2} \\
\lambda_{k}>\delta h_{k}, t_{k} \geq T_{2} .
\end{array}\right.
$$

Substituting (3.17) into Inequality (3.14), we obtain

$$
\left\{\begin{array}{c}
\lambda(t) \geq \delta h \frac{q(t)}{q\left(t-\tau_{i}\right)} \prod_{\ell=1}^{m_{j}} \sum_{i=1}^{m} p_{i}^{\alpha_{\ell}}\left(t-\sigma_{\ell}\right) \exp \left(\delta h \tau_{i}\right)(1-\mu)^{-\tau_{i}}+ \\
+q(t) \exp \left(\delta h \sum_{\ell=1}^{m_{j}} \alpha_{\ell} \sigma_{\ell}\right)(1-\mu)^{-\sigma_{\ell}} \\
\lambda_{k} \geq \delta h_{k} \frac{q_{k}}{q\left(t_{k}-\tau_{i}\right)} \prod_{\ell=1}^{m_{j}} \sum_{i=1}^{m} p_{i}^{\alpha_{\ell}}\left(t_{k}-\sigma_{\ell}\right) \exp \left(\delta h_{k} \tau_{i}\right)(1-\mu)^{-\tau_{i}}+ \\
+q_{k} \exp \left(\delta h_{k} \sum_{\ell=1}^{m_{j}} \alpha_{\ell} \sigma_{\ell}\right)(1-\mu)^{-\sigma_{\ell}}
\end{array}\right.
$$

for $t \geq T_{2}+\zeta$ and for all $\mathrm{k}: t_{k} \geq T_{2}+\zeta$. Hence

$$
\left\{\begin{array}{c}
h \geq \lim _{t \rightarrow \infty} \inf \left\{\delta h \frac{q(t)}{q\left(t-\tau_{i}\right)} \prod_{\ell=1}^{m_{j}} \sum_{i=1}^{m} p_{i}^{\alpha_{\ell}}\left(t-\sigma_{\ell}\right) \exp \left(\delta h \tau_{i}\right)(1-\mu)^{-\tau_{i}}+\right. \\
\left.+q(t) \exp \left(\delta h \sum_{\ell=1}^{m_{j}} \alpha_{\ell} \sigma_{\ell}\right)(1-\mu)^{-\sigma_{\ell}}\right\} \\
h_{k} \geq \lim _{t_{k} \rightarrow \infty} \inf \left\{\delta h_{k} \frac{q_{k}}{q\left(t_{k}-\tau_{i}\right)} \prod_{\ell=1}^{m_{j}} \sum_{i=1}^{m} p_{i}^{\alpha_{\ell}}\left(t_{k}-\sigma_{\ell}\right) \exp \left(\delta h_{k} \tau_{i}\right)(1-\mu)^{-\tau_{i}}+\right. \\
\left.+q_{k} \exp \left(\delta h_{k} \sum_{\ell=1}^{m_{j}} \alpha_{\ell} \sigma_{\ell}\right)(1-\mu)^{-\sigma_{\ell}}\right\}
\end{array}\right.
$$

If we set $\lambda^{*}=\delta h$ and $\lambda_{k}^{*}=\delta h_{k}$, then

$$
\left\{\begin{aligned}
& \lambda^{*} \geq \delta \lim _{t \rightarrow \infty} \inf \left\{\lambda^{*} \frac{q(t)}{q\left(t-\tau_{i}\right)} \prod_{\ell=1}^{m_{j}} \sum_{i=1}^{m} p_{i}^{\alpha_{\ell}}\left(t-\sigma_{\ell}\right) \exp \left(\lambda^{*} \tau_{i}\right)(1-\mu)^{-\tau_{i}}+\right. \\
&\left.+q(t) \exp \left(\lambda^{*} \sum_{\ell=1}^{m_{j}} \alpha_{\ell} \sigma_{\ell}\right)(1-\mu)^{-\sigma_{\ell}}\right\} \\
& \lambda_{k}^{*} \geq \delta \lim _{t_{k} \rightarrow \infty} \inf \left\{\lambda_{k}^{*} \frac{q_{k}}{q\left(t_{k}-\tau_{i}\right)} \prod_{\ell=1}^{m_{j}} \sum_{i=1}^{m} p_{i}^{\alpha_{\ell}}\left(t_{k}-\sigma_{\ell}\right) \exp \left(\lambda_{k}^{*} \tau_{i}\right)(1-\mu)^{-\tau_{i}}+\right. \\
&\left.+q_{k} \exp \left(\lambda_{k}^{*} \sum_{\ell=1}^{m_{j}} \alpha_{\ell} \sigma_{\ell}\right)(1-\mu)^{-\sigma_{\ell}}\right\}
\end{aligned}\right.
$$


which comes into contradiction with Inequality (3.16). This completes the proof of Theorem 3.1 under Inequality (3.11). If Condition (3.12) holds, we set $\lambda(t) q(t)=-\frac{z^{\prime}(t)}{z(t)}$ and $\lambda_{k} q_{k}=-\frac{\Delta z\left(t_{k}\right)}{z\left(t_{k}\right)}$. Then Equation (3.13) becomes

$$
\left\{\begin{array}{r}
\lambda(t) \geq \lambda\left(t-\tau_{i}\right) \prod_{\ell=1}^{m_{j}} \sum_{i=1}^{m} p_{i}^{\alpha_{\ell}}\left(t-\sigma_{\ell}\right) \exp \left(\int_{t-\tau_{i}}^{t} \lambda(s) q(s) d s\right) \prod_{t-\tau_{i} \leq t_{j}<t}\left(1-(\lambda q)_{j}\right)^{-1}+ \\
+q(t) \exp \left(\sum_{\ell=1}^{m_{j}} \alpha_{\ell} \int_{t-\sigma_{\ell}}^{t} \lambda(s) q(s) d s\right) \prod_{t-\sigma_{\ell} \leq t_{j}<t}\left(1-(\lambda q)_{j}\right)^{-1} \\
\lambda_{k} \geq \lambda\left(t_{k}-\tau_{i}\right) \prod_{\ell=1}^{m_{j}} \sum_{i=1}^{m} p_{i}^{\alpha_{\ell}}\left(t_{k}-\sigma_{\ell}\right) \exp \left(\int_{t_{k}-\tau_{i}}^{t_{k}} \lambda(s) q(s) d s\right) \prod_{t_{k}-\tau_{i} \leq t_{j}<t_{k}}\left(1-(\lambda q)_{j}\right)^{-1}+ \\
+q_{k} \exp \left(\sum_{\ell=1}^{m_{j}} \alpha_{\ell} \int_{t_{k}-\sigma_{\ell}}^{t_{k}} \lambda(s) q(s) d s\right) \prod_{t_{k}-\sigma_{\ell} \leq t_{j}<t_{k}}\left(1-(\lambda q)_{j}\right)^{-1}
\end{array}\right.
$$

By Lemma 3.2, we know that

$$
\left\{\begin{array}{l}
\lim _{t \rightarrow \infty} \inf \int_{t-\sigma^{*}}^{t} \lambda(s) q(s) d s<\infty \\
\lim _{t_{k} \rightarrow \infty} \inf \int_{t_{k}-\sigma^{*}}^{t_{k}} \lambda(s) q(s) d s<\infty .
\end{array}\right.
$$

Therefore, using Inqualities (3.6) and (3.19), we conclude that

$$
\left\{\begin{array}{l}
\lim _{t \rightarrow \infty} \inf \lambda(t)<\infty \\
\lim _{t_{k} \rightarrow \infty} \inf \lambda_{k}<\infty
\end{array}\right.
$$

From Inequality (3.18), $\lambda(t) \geq 1, \lambda_{k} \geq 1$ and hence

$$
\left\{\begin{array}{l}
0<\lim _{t \rightarrow \infty} \inf \lambda(t)=\lambda<\infty \\
0<\lim _{t_{k} \rightarrow \infty} \inf \lambda\left(t_{k}\right)=\lambda_{k}<\infty .
\end{array}\right.
$$

Thus, by virtue of Inequality (3.12), there exists $\delta \in(0,1)$ such that

$$
\left\{\begin{array}{c}
\delta \liminf _{t \rightarrow \infty}\left\{\operatorname { i n f } _ { \lambda > 0 } \left[\prod_{\ell=1}^{m_{j}} \sum_{i=1}^{m} p_{i}^{\alpha_{\ell}}\left(t-\sigma_{\ell}\right) \exp \left(\lambda \int_{t-\tau_{i}}^{t} q(s) d s\right)(1-\mu)^{-\tau_{i}}+\right.\right. \\
\left.\left.+\frac{1}{\lambda} \exp \left(\lambda \sum_{\ell=1}^{m_{j}} \alpha_{\ell} \int_{t-\sigma_{\ell}}^{t} q(s) d s\right)(1-\mu)^{-\sigma_{\ell}}\right]\right\}>1 \\
\delta \lim _{t_{k} \rightarrow \infty} \inf \left\{\operatorname { i n f } _ { \lambda _ { k } > 0 } \left[\prod_{\ell=1}^{m_{j}} \sum_{i=1}^{m} p_{i}^{\alpha_{\ell}}\left(t_{k}-\sigma_{\ell}\right) \exp \left(\lambda_{k} \int_{t_{k}-\tau_{i}}^{t_{k}} q(s) d s\right)(1-\mu)^{-\tau_{i}}+\right.\right. \\
\left.\left.+\frac{1}{\lambda_{k}} \exp \left(\lambda_{k} \sum_{\ell=1}^{m_{j}} \alpha_{\ell} \int_{t_{k}-\sigma_{\ell}}^{t_{k}} q(s) d s\right)(1-\mu)^{-\sigma_{\ell}}\right]\right\}>1
\end{array}\right.
$$

where $\mu=(\lambda q)_{j}$. Using a reasoning analogous to that given in the proof of Inequality (3.16), we reach a contradiction. This completes the proof of Theorem 3.1.

Corollary 3.1 Assume the fulfilment of Conditions C3.1 - C3.4 and Inequality (3.6) with

$$
\left\{\begin{array}{c}
\liminf _{t \rightarrow \infty}\left\{\operatorname { i n f } _ { \lambda > 0 } \left[\sum_{i=1}^{m} p_{i}(t-\sigma) \frac{q(t)}{q\left(t-\tau_{i}\right)} \exp \left(\lambda \tau_{i}\right)\left(1-\lambda_{j}\right)^{-\tau_{i}}+\right.\right. \\
\left.\left.+\frac{q(t)}{\lambda} \exp (\lambda \sigma)\left(1-\lambda_{j}\right)^{-\sigma}\right]\right\}>1
\end{array}\right.
$$

Then every solution of equation (3.2) is oscillatory.

\section{References}

D. D. Bainov and P. S. Simeonov. (1998). Oscillation Theory of Impulsive Differential Equations, International Publications. Orlando, Florida. 
D. V. Giang and I. Gyori. (1993). Oscillation of a linear neutral delay differential equation with unbounded time lag, Differential Equations and Dynamical Systems, 14, 267 - 274.

H. A. El-Morshedy and K. Gopalsamy, Nonoscillation. (2000). oscillation and convergence of a class of neutral equations, Nonlinear Anal. Theor. Meth. Appl., 40, 173 - 183.

I. O. Isaac and Z. Lipcsey. (2009a). Linearized Oscillations in Nonlinear Neutral Delay Impulsive Impulsive Differential Equations, Journal of Modern Mathematics and Statistics, 3, 1 - 7.

I. O. Isaac and Z. Lipcsey. (2009b). Oscillations in Systems of Neutral Impulsive Differential Equations, Journal of Modern Mathematics and Statistics, 3, 17 - 21.

I. O. Isaac and Z. Lipcsey. (2009c). Oscillations in Non-autonomous Neutral Impulsive Differential Equations with Several Delays, Journal of Modern Mathematics and Statistics, 3, 73 - 77.

I. O. Isaac and Z. Lipcsey. (2010a). Oscillations in Linear Neutral Delay Impulsive Differential Equations with Constant Coefficients, Communications in Applied Analysis, 14, 123 - 136.

I. O. Isaac and Z. Lipcsey. (2010b). Oscillations in Neutral Impulsive Differential Equations with variable Coefficients, Dynamic Systems and Applications, 19, 45 - 62.

J. R. Graef, J. H. Shen and I. P. Stavroulakis. (2002). Oscillation of impulsive neutral delay differential equations, J. Math. Anal. Appl., 268, $310-333$.

J. R. Graef, R. Savithri and E. Thandapani. (2004). Oscillation of first order neutral delay differential equations, Electron. J. Qual. Theory Diff. Equ., 12, 1 - 11 .

L. Berezansky and E. Braverman. (1996). Oscillation of linear delay impulsive differential equations, Comm. Appl. Nonlinear Anal. Theor. Meth. Appl., 3 , 61-77.

L. Zhiguo, L. Xiaoyan and S. Jianhua. (2000). Oscillation of impulsive neutral differential equations with positive and negative coefficients, Indian J. Pure Appl. Math, 31, 753-766. 